\title{
The search for putative unifying genetic factors for components of the metabolic syndrome
}

\author{
M. Sjögren • V. Lyssenko • A. Jonsson • G. Berglund • \\ P. Nilsson • L. Groop • M. Orho-Melander
}

Received: 5 June 2008 / Accepted: 20 August 2008 / Published online: 14 October 2008

(C) Springer-Verlag 2008

\begin{abstract}
Aims/hypothesis The metabolic syndrome is a cluster of factors contributing to increased risk of cardiovascular disease and type 2 diabetes but unifying mechanisms have not been identified. Our aim was to study whether common variations in 17 genes previously associated with type 2 diabetes or components of the metabolic syndrome and variants in nine genes with inconsistent association with at least two components of the metabolic syndrome would also predict future development of components of the metabolic syndrome, individually or in combination.

Methods Genetic variants were studied in a large prospective study of 16,143 non-diabetic individuals (mean followup time 23 years) from the Malmö Preventive Project. In this study, development of at least three of obesity (BMI $\geq$ $30 \mathrm{~kg} / \mathrm{m}^{2}$ ), dyslipidaemia (triacylglycerol $\geq 1.7 \mathrm{mmol} / \mathrm{l}$ and/
\end{abstract}

Electronic supplementary material The online version of this article (doi:10.1007/s00125-008-1151-4) contains supplementary material, which is available to authorised users.

M. Sjögren $(\bowtie) \cdot$ V. Lyssenko • A. Jonsson • L. Groop •

M. Orho-Melander

Department of Clinical Sciences, Diabetes and Endocrinology, Lund University,

CRC house 91, floor 12, UMAS entrance 72,

20502 Malmö, Sweden

e-mail: marketa.sjogren@med.lu.se

G. Berglund · P. Nilsson

Department of Clinical Sciences, Medicine, Lund University, Malmö, Sweden

\section{Groop}

Department of Medicine, Helsinki University Central Hospital and Research Program of Molecular Medicine, University of Helsinki,

Helsinki, Finland or lipid-lowering treatment), hypertension (blood pressure $\geq$ $140 / 90 \mathrm{mmHg}$ and/or antihypertensive medication) and hyperglycaemia (fasting plasma glucose $\geq 5.6 \mathrm{mmol} / \mathrm{l}$ and/or known diabetes) was defined as development of the metabolic syndrome. The risk of developing at least three components of the metabolic syndrome or the individual components was calculated by logistic regression adjusted for age at baseline, follow-up time and sex.

Results Polymorphisms in TCF7L2 (rs7903146, OR 1.10, 95\% CI 1.04-1.17, $p=0.00097$ ), FTO (rs9939609, OR 1.08, 95\% CI 1.02-1.14, $p=0.0065$ ), WFS1 (rs10010131, OR $1.07,95 \%$ CI $1.02-1.13, p=0.0078)$ and $I G F 2 B P 2$ (rs4402960, OR 1.07, 95\% CI 1.01-1.13, $p=0.021$ ) predicted the development of at least three components of the metabolic syndrome in both univariate and multivariate analysis; in the case of TCF7L2, WFS1 and IGF2BP this was due to their association with hyperglycaemia $(p<$ $0.00001, p=0.0033$ and $p=0.027$, respectively) and for FTO it was due to its association with obesity $(p=0.004)$. A polymorphism in the GCKR gene predicted dyslipidaemia (rs1260326, OR 1.15, 95\% CI 1.09-1.22, $p<0.00001$ ) but not the metabolic syndrome. None of the studied polymorphisms was associated with more than two components of the metabolic syndrome. A composite genotype score of the 17 polymorphisms associated with type 2 diabetes predicted the development of at least three components of the metabolic syndrome (OR 1.04, $p<0.00001)$ and the development of hyperglycaemia (OR 1.06, $p<0.00001$ ). Carriers of $\geq 19$ risk alleles had 51 and $72 \%$ increased risk of developing at least three components of the metabolic syndrome and hyperglycaemia, respectively, compared with carriers of $\leq 12$ risk alleles ( $p<0.00001$ for both).

Conclusions/interpretation Polymorphisms in susceptibility genes for type 2 diabetes (TCF7L2,WFS1, IGF2BP2) and obesity $(F T O)$ predispose to the metabolic syndrome by 
increasing the risk of one specific component of the metabolic syndrome. The findings argue against a unifying genetic component for the metabolic syndrome.

Keywords Common polymorphism · Genetics · Metabolic syndrome $\cdot$ Prospective study Type 2 diabetes

$\begin{array}{ll}\text { Abbreviations } \\ \text { CVD } & \text { cardiovascular disease } \\ \text { GWAS } & \text { genome-wide association study } \\ \text { IDF } & \text { International Diabetes Federation } \\ \text { MMP } & \text { Malmö Preventive Project } \\ \text { NCEP- } & \text { National Cholesterol Education Program } \\ \text { ATPIII } & \text { Adult Treatment Panel III } \\ \text { SNP } & \text { single nucleotide polymorphism }\end{array}$

\section{Introduction}

The metabolic syndrome is defined as a cluster of factors that increase the risk of type 2 diabetes and cardiovascular disease (CVD) [1]. In addition to environmental factors, such as a sedentary lifestyle and an energy-rich diet, substantial evidence exists for a genetic component of the syndrome. Environmental triggers may thus play a variable role depending on the genetic background of the individual. The metabolic syndrome clusters in families [2] and its prevalence differs between ethnic groups [3]. The heritability of the metabolic syndrome has been estimated to be about 24\% [4] and that of components of the metabolic syndrome ranges from $\sim 16 \%$ for systolic blood pressure to $\sim 60 \%$ for HDL-cholesterol levels [4-7].

It is not established whether the metabolic syndrome has a common pathogenic background. One way to address this question is to study whether the different components of the metabolic syndrome share associated genetic factors.

To accomplish this, we first investigated whether common variants in genes that have previously been associated with type 2 diabetes in candidate gene or linkage studies and convincingly replicated (TCF7L2, PPARG, KCNJ11 and WFS1) or have been identified in genomewide association studies (GWAS) or meta-analysis of GWAS with genome-wide significant $p$ values (SLC30A8, HHEX, IGF2BP2, CDKAL1, CDKN2A, FTO, JAZF1, CDC123/CAMK1D, TSPAN8/LGR5, THADA, ADAMTS9, NOTCH2 and GCKR) [8-18] also predict the development of components of the metabolic syndrome, individually or in combination, in 16,143 individuals from the Malmö Preventive Project (MPP) followed for a mean period of 23 years. Second, we investigated ten polymorphisms in nine genes (ADRB1, ADRB2, ADRB3, CAPN10, IRS1,
UCP2, PPARGC1A, PTPN1 and ENPP1), each of which has been associated with at least two components contributing to the metabolic syndrome [19-32].

\section{Methods}

In the MPP, 33,346 Swedish participants $(22,444$ men and 10,902 women, mean age 49 years, $24.5 \%$ with IFG and/ or IGT from the city of Malmö in southern Sweden participated in health screening during 1974-1992 (attendance rate $71 \%$ ). All individuals underwent a physical examination and measurements of fasting blood glucose and triacylglycerol concentrations were performed. In addition, 18,900 consecutive individuals also underwent an OGTT. Information on lifestyle factors and medical history was obtained from a questionnaire. Of individuals participating in the initial screening, 4931 have died and 551 were lost from follow-up for other reasons. Twentyfive thousand of the eligible individuals were invited to a re-screening visit during 2002-2006, including a physical examination and fasting blood samples for measurements of glucose, triacylglycerol and HDL-cholesterol concentrations. Of the invited individuals, 17,284 participated in the re-screening; 1,141 of them were excluded from the present study because of the lack of DNA or crucial clinical information or because of type 2 diabetes at baseline. Thus, 16,143 non-diabetic participants, 2,063 of whom developed type 2 diabetes, were included in the follow-up study [33]. The diagnosis of diabetes was confirmed from patient records or was based on a fasting plasma glucose concentration greater than $7.0 \mathrm{mmol} / \mathrm{l}$. The clinical characteristics of individuals included in the study both at baseline and at follow-up are summarised in Table 1.

Definition of the metabolic syndrome As waist and HDLcholesterol measurements were not available for most participants at baseline, we were unable to construct the definitions of the metabolic syndrome according to any of the common definitions (National Cholesterol Education Program Adult Treatment Panel III [NCEP-ATPIII] [34], WHO [35], International Diabetes Federation [IDF] [36]). We therefore defined the components of the metabolic syndrome as follows: (1) obesity $\left(\mathrm{BMI} \geq 30 \mathrm{~kg} / \mathrm{m}^{2}\right)$; (2) dyslipidaemia (triacylglycerol $\geq 1.7 \mathrm{mmol} / 1$ and/or lipidlowering treatment); (3) hypertension (blood pressure $\geq$ $140 / 90 \mathrm{mmHg}$ and/or antihypertensive medication); and (4) hyperglycaemia (fasting plasma glucose $\geq 5.6 \mathrm{mmol} / 1$ and/or overt diabetes). The presence of at least three of these components defined the metabolic syndrome and occurred in $6.7 \%$ individuals at baseline; $35 \%$ had none of the components. 
Table 1 Clinical characteristics of MPP participants at baseline and after follow-up

\begin{tabular}{lllll}
\hline Characteristic & Data available $(n)$ & Baseline characteristic & Data available $(n)$ & Re-examination characteristic \\
\hline Number of participants & 16,143 & & 16,143 & $10,455,5688$ \\
Men, women & $10,455,5688$ & & 16,143 & $68 \pm 6^{* * *}$ \\
Age (years) & 16,143 & $46 \pm 7$ & 16,055 & $27.1 \pm 4.1^{* * *}$ \\
BMI $\left(\mathrm{kg} / \mathrm{m}^{2}\right)$ & 16,136 & $24.3 \pm 3.3$ & 16,051 & $94.8 \pm 12.2$ \\
Waist (cm) & 827 & $78.6 \pm 9.8$ & 16,099 & $145 \pm 20^{* * *}$ \\
Systolic BP (mmHg) & 16,140 & $127 \pm 14$ & 16,099 & $84 \pm 11$ \\
Diastolic BP (mmHg) & 16140 & $84 \pm 9$ & 16,111 & $5.8 \pm 1.3^{* * *}$ \\
Fasting plasma glucose (mmol/1) & 16,083 & $5.4 \pm 0.6$ & 16,118 & $1.26 \pm 0.80$ \\
Triacylglycerol (mmol/1) & 16,104 & $1.26 \pm 0.77$ & 16,112 & $1.40 \pm 0.42$ \\
HDL-cholesterol (mmol/1) & 289 & $1.54 \pm 0.37$ & 0 & $\mathrm{NA}$ \\
Smoking (\%) & 15,736 & 37.3 & 16,141 & $37.7^{* * *}$ \\
Antihypertensive medication (\%) & 16,119 & 4.2 & 16,141 & 20.0 \\
Lipid-lowering medication (\%) & 0 & $\mathrm{NA}$ & 16,122 & $13.2^{* * * *}$ \\
Type 2 diabetes (\%) & 16,143 & 0 & 16,042 & $21.2^{* * *}$ \\
Obesity (\%) & 16,135 & 5.1 & 16,118 & $34.5^{* * *}$ \\
Dyslipidaemia (\%) & 16,104 & 18.2 & 16,099 & $84.5^{* * *}$ \\
Hypertension (\%) & 16,124 & 34.2 & 16,117 & $47.2^{* * *}$ \\
Hyperglycaemia (\%) & 16,143 & 39.9 & 16,080 & $28.3^{* * *}$ \\
Metabolic syndrome & 16,131 & 6.7 & 16,080 & 30.2 \\
Metabolic syndrome, NCEP-ATPIII & 0 & $\mathrm{NA}$ & \\
\hline
\end{tabular}

Data are mean \pm SD

For the definition of the metabolic syndrome used in this study, please refer to the Methods $* * * p<0.001$

${ }^{a}$ Refers both to current and previous smoking

NA, not available

SNP selection and genotyping We selected 17 polymorphisms associated with type 2 diabetes to be investigated in this study. Four of the selected single nucleotide polymorphisms (SNPs) had been identified earlier in candidate gene or linkage studies and convincingly replicated (TCF7L2, PPARG, KCNJ11 and WFS1) [9, 11-13]. Seven of the SNPs were identified by GWAS and were genomewide significant (SLC30A8, HHEX, IGF2BP2, CDKAL1, $C D K N 2 A, F T O$ and GCKR) [14-17]. The remaining six SNPs were identified in a meta-analysis of GWAS (JAZF1, CDC123/CAMK1D, TSPAN8/LGR5, THADA, ADAMTS9 and $N O T C H 2)$ [8] at a genome-wide-significant level. Of them FTO has been associated with obesity and type 2 diabetes, whereas GCKR has been associated with increased triacylglycerol and decreased glucose levels [10, 14, 18, 37-39]. We additionally selected ten SNPs in nine genes (ADRB1, ADRB2, ADRB3, CAPN10, IRS1, UCP2, PPARGC1A, PTPN1 and ENPP1) that have been associated with at least two components of the metabolic syndrome, such as type 2 diabetes (ADRB2, ADRB3, CAPN10, IRS1, UCP2, PPARGC1A and ENPP1) [19, 23, 25, 29, 30, 40], obesity (ADRB1, ADRB2, ADRB3, CAPN10, IRS1, UCP2, PTPN1 and ENPP1 [21, 22, 26-29, 32, 41]), hypertension (ADRB1, PPARGC1A and PTPN1) [20, 27, 31] or the metabolic syndrome (ADRB1 and ADRB3) [42].
DNA was extracted from whole blood using Qiagen Maxipreps (Qiagen, Valencia, CA, USA). Genotyping was performed using matrix-assisted laser desorption ionisationtime of flight mass spectrometry on the MassARRAY platform (Sequenom, San Diego, CA, USA) (rs7903146, rs1801282, rs5219, rs7754840, rs10811661, rs1044498, rs3787348, rs8192678, rs1801278, rs659366, rs2975760 and rs3792267), by an allelic discrimination assay-bydesign method using an ABI 7900 PCR system (Applied Biosystems, Foster City, CA, USA) (rs4402960, rs9939609, rs10010131, rs1111875, rs864745, rs12779790, rs7961581, rs7578597, rs4607103, rs10923931, rs1260326, rs1801253, rs1042714 and rs4994) and using a Kaspar allelic discrimination method (KBioscience, Hoddesdon, UK) (rs13266634).

To ascertain genotype quality, random samples of 5\% were re-genotyped and the concordance rate was $>99 \%$. All SNPs were in Hardy-Weinberg equilibrium $(p>0.01)$ with the exception of rs864745 in the JAZF1 gene $(p=0.001)$. Genotyping errors are an unlikely explanation for this as 2416 samples $(15 \%)$ were re-genotyped with two methods (MassARRAY and ABI 7900) with a concordance of 98.7\%.

Statistical analyses The OR for the risk of developing the metabolic syndrome or its components was calculated using 
Table 2 Risk of developing at least three components of the metabolic syndrome

\begin{tabular}{llll}
\hline Gene symbol & SNP & OR $(95 \%$ CI $)$ & $p$ value \\
\hline TCF7L2 & rs7903146 & $1.10(1.04-1.17)$ & 0.00097 \\
PPARG & rs1801282 & $0.97(0.90-1.05)$ & 0.50 \\
KCNJ11 & rs5219 & $1.01(0.96-1.07)$ & 0.66 \\
HHEX & rs1111875 & $1.05(1.00-1.11)$ & 0.069 \\
IGF2BP2 & rs4402960 & $1.07(1.01-1.13)$ & 0.021 \\
CDKAL1 & rs7754840 & $0.97(0.91-1.03)$ & 0.33 \\
FTO & rs9939609 & $1.08(1.02-1.14)$ & 0.0065 \\
CDKN2A & rs10811661 & $1.07(1.00-1.15)$ & 0.059 \\
SLC30A8 & rs13266634 & $1.04(0.98-1.10)$ & 0.22 \\
WFS1 & rs10010131 & $1.07(1.02-1.13)$ & 0.0078 \\
GCKR & rs1260326 & $1.03(0.97-1.09)$ & 0.33 \\
JAZF1 & rs864745 & $1.00(0.95-1.06)$ & 0.88 \\
ADAMTS9 & rs4607103 & $1.00(0.94-1.06)$ & 0.87 \\
CAMK1D & rs12779790 & $1.00(0.93-1.07)$ & 0.89 \\
TSPAN8/LGR5 & rs7961581 & $0.98(0.93-1.04)$ & 0.57 \\
NOTCH2 & rs10923931 & $0.96(0.88-1.06)$ & 0.45 \\
THADA & rs7578597 & $1.05(0.96-1.14)$ & 0.33 \\
\hline
\end{tabular}

All analyses are adjusted for age at baseline, follow-up time and sex The $p$ values given are two-sided, uncorrected for multiple testing Proteins encoded by genes listed (in order of appearance in table): $T C F 7 L 2$, transcription factor 7-like 2; PPARG, peroxisome proliferator-activated receptor-gamma; $K C N J 11$, potassium channel, inwardly rectifying, subfamily $\mathrm{J}$, member $11, H H E X$, hematopoetically expressed homeobox; IGF2BP2, insulin-like growth factor 2 mRNAbinding protein $2, C D K A L 1, \mathrm{CDK} 5$ regulatory subunit-associated protein 1-like 1; FTO, fat mass- and obesity-associated; $C D K N 2 A$, cyclin-dependent kinase inhibitor 2A; SLC30A8, solute carrier family 30 (zinc transporter) member 8; WFS1, Wolfram syndrome 1; GCKR, glucokinase regulatory protein; $J A Z F 1$, juxtaposted with another zinc finger gene 1; ADAMTS9, a disintegrin-like and metalloproteinase with thrombospondin type 1 motif, 9; CAMK1D, calcium/calmodulindependent protein kinase 1-delta; TSPAN8, tetraspanin 8; LGR5, leucine-rich repeat-containing $\mathrm{G}$ protein-coupled receptor 5; NOTCH2, notch homolog 2; THADA, thyroid adenoma associated

logistic regression analyses assuming an additive model, adjusted for age at baseline, follow-up time and sex. Only participants free from the investigated component of the metabolic syndrome at baseline were included in the analysis. Multivariate logistic regression analyses were performed adjusting for age at baseline, follow-up time and sex and including all the 17 gene polymorphisms associated with type 2 diabetes in the model. In addition, we conducted backward elimination of SNPs with a retention threshold of $p<0.05$. The two methods gave similar results and only the backward elimination results are presented.

As all the individual SNPs were assumed to explain only a very small proportion of the individual or combined components of the metabolic syndrome, we constructed a composite genotype score on the basis of the number of unfavourable alleles (those associated with type 2 diabetes except in $G C K R$, where the unfavourable allele was that associated with higher triacylglycerol levels) that were carried by each individual for each of the 17 SNPs associated with type 2 diabetes. In addition, we constructed a composite risk score by including only SNPs significant for any components of the metabolic syndrome in the multivariate analysis (TCF7L2, HHEX, FTO, IGF2BP2, CDKAL1, SLC30A8 and GCKR).

All statistical analyses were performed using the SPSS 14.0 statistical analysis software package (SPSS, Chicago, IL, USA), and nominal two-sided $p$ values and corrected $p$ values (Bonferroni, corrected for 27 SNPs included in this study) of $p<0.05$ were considered significant. Power calculations were performed with the genetic power calculator at http://pngu.mgh.harvard.edu/ purcell/gpc/cc2. html [43]. In our sample of 14,996 individuals with fewer than three components of the metabolic syndrome at baseline, 3843 had at least three components of the syndrome (cases) and 11,153 had fewer than three components of the metabolic syndrome (controls) after follow-up time. The risk allele frequencies ranged from 0.1 to 0.9 and at the significance level of 0.05 our power to detect association was $76-99 \%$ for OR 1.1-1.2.

\section{Results}

Components of the metabolic syndrome in MPP at baseline and after the follow-up At inclusion in the study, 6.7\% of individuals had at least three components of the metabolic syndrome according to the criteria described above. Among individuals with fewer than three components of the metabolic syndrome at baseline, $25.6 \%$ had at least three components of the syndrome after the mean follow-up time of 23 years. Among individuals without a specific component of the metabolic syndrome at baseline, $18.0 \%$ developed obesity, $28.0 \%$ dyslipidaemia, $73.5 \%$ hypertension and $37.9 \%$ hyperglycaemia during the follow-up time. Compared with the baseline situation, in which $35.0 \%$ of the individuals had none of the four components of the metabolic syndrome, $8.7 \%$ had none at follow-up $(p<$ 0.00001 ), $30.1 \%$ had one (compared with $38.1 \%$ at baseline, $p<0.00001$ ), $32.0 \%$ had two (compared with $19.1 \%$ at baseline, $p<0.00001$ ), 20.9\% had three (compared with $5.8 \%$ at baseline, $p<0.00001$ ) and $7.3 \%$ had four (compared with $0.8 \%$ at baseline, $p<0.00001$ ) (Table 1). The risk of developing at least three of four components of the metabolic syndrome was affected by age at baseline (OR 0.992, 95\% CI 0.986-0.999, $p=0.016$ ), smoking (OR $1.36,95 \%$ CI 1.26-1.47, $p<0.00001)$ and male sex (OR $1.41,95 \%$ CI $1.27-1.57, p<0.00001)$, after adjustment for follow-up time. 
At follow-up, data were available to define the metabolic syndrome also using the NCEP-ATPIII criteria. In total, $30.2 \%$ of the study participants had the metabolic syndrome according to the NCEP-ATPIII definition compared with $28.8 \%$ having at least three of the four components of the metabolic syndrome available in this study, and $22.2 \%$ had the metabolic syndrome according to both definitions.

Genetic prediction of components of the metabolic syndrome in MPP Out of the 17 SNPs associated with type 2 diabetes, polymorphisms in TCF7L2 (rs7903146, OR $1.10,95 \%$ CI $1.04-1.17, p=0.00097)$, FTO (rs9939609, OR 1.08, 95\% CI 1.02-1.14, $p=0.0065$ ), WFS1 (rs10010131, OR 1.07, 95\% CI 1.02-1.13, $p=0.0078$ ) and IGF2BP2 (rs4402960, OR 1.07, 95\% CI 1.01-1.13, $p=0.021)$ predicted the presence of at least three of the four components of the metabolic syndrome after the follow-up time (Table 2). However, after correction for multiple testing, only the TCF7L2 rs7903146 remained significant $\left(p_{\mathrm{c}}=0.026\right)$. In multivariate logistic regression analysis, the following three SNPs were retained as predictors of at least three components of the metabolic syndrome present in an individual after follow-up: rs7903146 in TCF7L2 $(p=0.0012)$, rs111875 in HHEX $(p=0.0052)$ and rs9939609 in FTO $(p=0.018)$.

Notably, for the variants in TCF7L2 (rs7903146, OR $1.17,95 \%$ CI $1.09-1.25, p<0.00001), I G F 2 B P 2$ (rs4402960, OR 1.10, 95\% CI 1.03-1.18, $p=0.0033$ ), CDKAL1 (rs7754840, OR 1.12, 95\% CI 1.04-1.20, $p=0.0017)$, SLC30A8 (rs13266634, OR 1.12, 95\% CI $1.05-1.19, p=0.00061)$, WFS1 (rs10010131, OR 1.07, $95 \%$ CI $1.01-1.14, p=0.027$ ) the risk of development of at least three of the four components of the metabolic syndrome could be explained by their effect on hyperglycaemia. The same four SNPs were retained as predictors of hyperglycaemia after follow-up in multivariate regression analysis (rs7903146, $p<0.00001$; rs4402960, $p=0.025$; rs7754840, $p=0.0019$; and rs13266634, $p=0.0037)$. Even after correction for multiple testing, variants of $T C F 7 L 2$ and $C D K A L 1$ remained significant predictors of hyperglycaemia $\left(p_{\mathrm{c}}=0.00016\right.$ and 0.032 , respectively).

The risk of developing obesity was increased by the polymorphism in FTO (rs9939609, OR 1.09, 95\% CI 1.03$1.16, p=0.004$ ) but the SNP did not remain significant after correction for multiple testing. In multivariate regression analysis, none of the SNPs was retained as a significant predictor of obesity at follow-up.

The risk of developing dyslipidaemia increased in individuals carrying the $\mathrm{T}$ allele of GCKR rs 1260326 both in univariate (OR 1.15, 95\% CI 1.09-1.22, $p<0.00001$, $\left.p_{\mathrm{c}}=0.000053\right)$ and multivariate analysis $(p<0.00001$ for
$G C K R$ ); no other variant increased the risk of dyslipidaemia (Table 3).

In multivariate regression analysis the rs13266634 in SLC30A8 $(p=0.014)$ was retained as a predictor of hypertension at follow-up, the $\mathrm{C}$ allele previously associated with hyperglycaemia now showing modestly reduced risk of developing hypertension (OR 0.88, 95\% CI 0.79-0.97).

Complete data for all 17 SNPs associated with type 2 diabetes were available for 9740 individuals and for the seven SNPs significant in multivariate regression analysis for 10,875 individuals. A composite genotype score of the 17 SNPs predicted the development of at least three components of the metabolic syndrome with $4 \%$ risk increase per allele (OR $1.04,95 \%$ CI $1.02-1.06, p<0.00001$ ) as well as the development of hyperglycaemia (OR $1.06,95 \%$ CI 1.04 $1.08, p<0.00001)$. Carriers of $\geq 19$ risk alleles $(9.7 \%$ of individuals) had $51 \%$ increased risk of developing at least three components of the metabolic syndrome (OR 1.51, 95\% CI 1.26-1.82, $p=0.000012)$ compared with individuals carrying $\leq 12$ risk alleles ( $15 \%$ of individuals) as well as 72 and $47 \%$ increased risk of developing hyperglycaemia (OR $1.72,95 \%$ CI 1.39-2.13, $p<0.00001$ ) and hypertension (OR $1.47,95 \%$ CI $1.11-1.96, p=0.0079)$, respectively.

When constructing a composite risk score by including only SNPs significant in the multivariate analysis $(T C F 7 L 2$, HHEX, FTO, IGF2BP2, CDKAL1, SLC3OA8 and GCKR) the carriers of $\geq 9$ risk alleles ( $6.7 \%$ of individuals) had increased risk of developing at least three components of the metabolic syndrome (OR $1.73,95 \%$ CI $1.39-2.15$, $p<0.00001$ ), hyperglycaemia (OR $1.97,95 \%$ CI $1.52-2.55$, $p<0.00001)$ and dyslipidaemia (OR $1.46,95 \%$ CI 1.16 $1.83, p=0.0011)$ compared with individuals carrying $\leq 3$ risk alleles ( $8.9 \%$ of individuals).

Although none of the ten SNPs in nine genes previously associated with components of the metabolic syndrome significantly predicted the development of at least three components of the syndrome in this study (see Electronic supplementary material Table 1), the Trp64Arg polymorphism in $A D R B 3$ nominally predicted the development of obesity (rs4994, OR 1.13, 95\% CI 1.01-1.26, $p=0.034$ ), whereas the SNP rs 1044498 in ENPP1 nominally predicted the development of hyperglycaemia (OR 1.10, 95\% CI $1.01-1.19, p=0.037)$. However, these results would not stand correction for multiple testing but replicate previous similar findings.

None of the studied polymorphisms was associated with more than two components of the metabolic syndrome.

\section{Discussion}

Our study addressed the question whether previously reported susceptibility genes for type 2 diabetes (TCF7L2, 


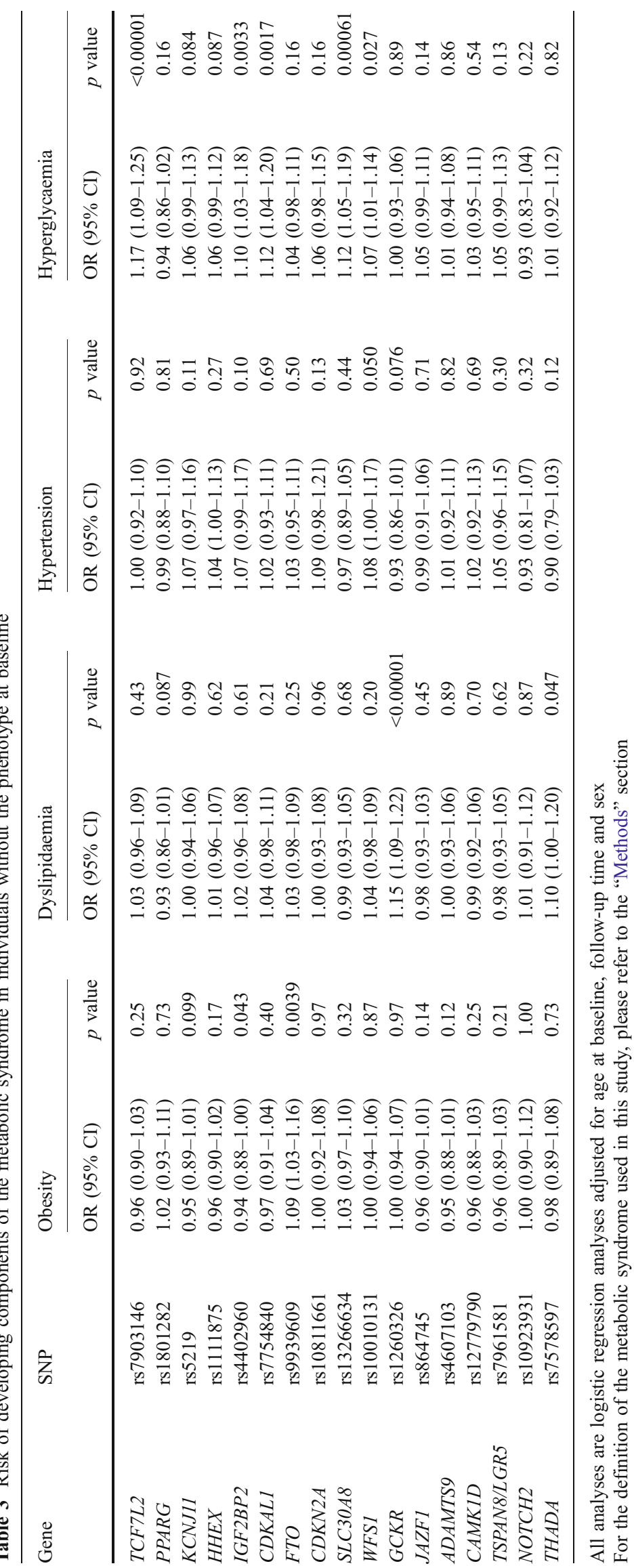


SLC30A8, HHEX, PPARG, KCNJ11, IGF2BP2, CDKAL1, CDKN2A, FTO, WFS1, JAZF1, CDC123/CAMK1D, TSPAN8/LGR5, THADA, ADAMTS9, NOTCH2 and $G C K R)$, obesity $(F T O)$ or dyslipidaemia $(G C K R)$ predict the development of components of the metabolic syndrome, individually or in combination, in a large prospective study with $>400,000$ follow-up years. Another key question was whether the different components would share a unique genetic factor. This was clearly not the case. Although a number of variants increased future risk of the metabolic syndrome, this could be explained by their effect on hyperglycaemia (TCF7L2, IGF2BP2 and WFS1) or obesity $(F T O)$. In Danish population-based twin studies, Benyamin et al. found only modest genetic correlations between the different components of the metabolic syndrome [44]. This led the authors to conclude that components of the metabolic syndrome did not share a major common genetic background, supporting our results.

The $\mathrm{T}$ allele of TCF7L2 rs7903146 was a strong predictor of the development of hyperglycaemia but was not associated with any of the other metabolic syndrome components. This is compatible with previous findings that variation in TCF7L2 influences insulin secretion but not insulin action [45].

Somewhat surprisingly, several of the variants (HHEX, PPARG, KCNJ11, FTO, NOTCH2 and JAZF1) which predict future type 2 diabetes in this cohort [33] did not predict the development of hyperglycaemia. This could be due to the low cut-off value used to define hyperglycaemia in this study as well as in the IDF definition of the metabolic syndrome, i.e. fasting plasma glucose $\geq 5.6 \mathrm{mmol} / \mathrm{l}$, or reduced power, as fewer individuals were included in the analysis than when analysing the whole cohort for prediction of type 2 diabetes $[36,46]$.

Although none of the polymorphisms previously inconsistently associated with components of the metabolic syndrome predicted the development of at least three components of the syndrome in our study, some results are in line with earlier reports; for example, the SNP rs4994 in the ADRB3 gene was weakly associated with the risk of developing obesity $(p=0.034)$ and the ENPP1 rs 1044498 was associated with a modestly increased risk of developing hyperglycaemia $(p=0.037)$. In our previous family-based study and in a large metaanalysis the C-allele (Arg) carriers of rs4994 had higher BMI than non-carriers [22, 32]. Association between ENPP1 and hyperglycaemia was supported by a recent meta-analysis indicating that the minor $\mathrm{C}$ allele increased the risk of future type 2 diabetes [25].

Two of the investigated polymorphisms, namely those in FTO and GCKR, have previously been convincingly associated with two components of the metabolic syndrome [10, 14, 18, 37-39]. Common variants in the FTO gene have been associated with both BMI and type 2 diabetes; the predisposition to type 2 diabetes can be fully explained by the weight-increasing effect [10]. In keeping with these findings, we observed that FTO predisposes to the metabolic syndrome predominantly via its effect on obesity. Although the mechanisms by which variants in FTO increase the risk of obesity are not known, they could involve effects on appetite regulation as FTO is strongly expressed in the hypothalamus $[47,48]$. In this study we did not observe the $F T O$ polymorphism as a significant risk factor for hyperglycaemia. This might again be due to reduced power when analysing fewer individuals or the relatively low cut-off value used to define hyperglycaemia.

The GCKR polymorphism has been associated primarily with triacylglycerol levels [14, 37, 38]. Interestingly, the same allele that has been associated with higher triacylglycerol levels has been associated with lower levels of fasting glucose [37-39]. In this study the GCKR variant did not predict hyperglycaemia or a combination of at least three components of the metabolic syndrome. The lack of association with hyperglycaemia might be due to reduced power, as we, in line with commonly used definitions of the metabolic syndrome, dichotomised the individuals as either being or not being hyperglycaemic. Indeed, the fasting plasma glucose levels were significantly lower in carriers of the $\mathrm{T}$ allele $(p=0.0025)$ at follow-up. The lack of association of the GCKR variant with at least three components of the metabolic syndrome is not surprising as the $\mathrm{T}$ allele is associated with higher triacylglycerol levels but also with lower glucose, a favourable metabolic marker. The risk of developing the metabolic syndrome might thus be balanced by these opposing associations.

The SNP rs13266634 in SLC30A8 was the only polymorphism associated with more than one component of the metabolic syndrome in multivariate logistic regression. As expected, association was found with hyperglycaemia, but the same allele was found to be slightly protective against the development of hypertension ( $p=$ $0.014)$. The mechanism for this observation is unclear and the modest $p$ value could imply a chance finding and needs confirmation in other studies.

Several studies have tried to identify underlying genetic risk factors for the metabolic syndrome [7], but no study has been able to identify genetic variants that would be shared by most components of the syndrome. Our relatively well-powered study showed that none of the genetic variants was shared by more than two of the components, thereby challenging the view that the metabolic syndrome has a common genetic background.

Despite its large size and long follow-up period, some limitations of the study should be mentioned. First, the definition of the metabolic syndrome is clearly known to influence both prevalence estimates and possible genetic 
associations [49]. The definition of obesity in most of the metabolic syndrome definitions uses measures of abdominal obesity, i.e. waist or waist-to-hip ratio instead of BMI. Although BMI and waist circumference correlate strongly, BMI captures only about $80 \%$ of the variance in waist circumference. Second, we lack data on HDL-cholesterol at baseline. At follow-up the prevalence of the metabolic syndrome in this cohort was slightly higher using the NCEP-ATPIII definition than using our modified definition without HDL-cholesterol $(30.2 \%$ vs $28.3 \%, p=0.00022)$. Including HDL-cholesterol in the definition of the metabolic syndrome classified another 1586 individuals as being dyslipidaemic at follow-up. Third, it is likely that we have underestimated the number of individuals who developed the metabolic syndrome as we can assume over-representation of the syndrome among participants who had died from cardiovascular disease.

One further caveat is that we restricted the analysis to known candidate genes. We cannot exclude the possibility that the different components would share some unknown genetic factor, which could only be identified by GWAS. It needs to be kept in mind that despite the recent success in finding genes contributing to complex metabolic traits defined as components of the syndrome (type 2 diabetes, obesity and lipid levels), no GWAS analysis for the metabolic syndrome has been reported. In addition, in GWAS for type 2 diabetes and obesity, the case groups have been selected mainly or solely on the basis of glucose tolerance status and BMI, respectively, and in none of the studies have individuals carrying other components of the metabolic syndrome than type 2 diabetes or obesity been excluded among controls, lowering the chance of identifying genes important for several of the components of the metabolic syndrome.

Although only seven of the 17 genes previously associated with type 2 diabetes predicted one of the components of the metabolic syndrome and only four predicted the development of at least three components of the syndrome in our study, a composite score including all 17 genes was significantly associated with the development of at least three components of the metabolic syndrome, though with a modest OR of 1.04 per allele. Nevertheless, individuals carrying at least 19 risk alleles had a more than $50 \%$ increased risk of having at least three components of the metabolic syndrome at follow-up compared with individuals with fewer than 13 risk alleles. However, as our study mainly focused on genes identified and verified as type 2 diabetes genes, it is obvious that the specific set of SNPs used to define the composite score for prediction of the metabolic syndrome in this study needs refinement. Because of the rapid progress of genome wide association and sequencing approaches, the flow of novel discoveries is expected to continue. In addition to the obvious need for
GWAS for the metabolic syndrome, new genes robustly associating with components of the metabolic syndrome are constantly emerging, such as $M C 4 R$ associated with obesity $[50,51]$ and the many novel genes regulating lipid levels [52-54]. We cannot exclude the possibility that one or several of these new candidate genes might provide a unifying genetic component for the metabolic syndrome.

We conclude that polymorphisms in candidate genes for type 2 diabetes (TCF7L2, WFS1, IGF2BP2) and obesity $(F T O)$ predict the development of at least three components of the metabolic syndrome by increasing the risk of development of the component of the metabolic syndrome for which the original association was reported. These data do not support the view that the different components of the metabolic syndrome share a common genetic background.

Acknowledgements This study was supported by the Novo Nordisk Foundation, the Swedish Medical Research Council, including Linné grant (No. 31475113580), the Knud and Alice Wallenberg Foundation, the Swedish Heart and Lung Foundation, the Region Skåne, the Medical Faculty of Lund University, Malmö University Hospital, the Albert Påhlsson Research Foundation, the Crafoord Foundation and the European Foundation for the Study of Diabetes. The authors are indebted to the staff and participants of the study population for their important contributions. We thank M. Svensson for technical assistance in Malmö and the RSKC Malmö (Region Skåne) genotyping facility for help with Sequenom genotyping.

Duality of interest The authors declare that there is no duality of interest associated with this manuscript.

\section{References}

1. Isomaa B, Almgren P, Tuomi $\mathrm{T}$ et al (2001) Cardiovascular morbidity and mortality associated with the metabolic syndrome. Diabetes Care 24:683-689

2. Bosy-Westphal A, Onur S, Geisler C et al (2007) Common familial influences on clustering of metabolic syndrome traits with central obesity and insulin resistance: the Kiel obesity prevention study. Int J Obes (Lond) 31:784-790

3. Cameron AJ, Shaw JE, Zimmet PZ (2004) The metabolic syndrome: prevalence in worldwide populations. Endocrinol Metab Clin North Am 33:351-375

4. Lin HF, Boden-Albala B, Juo SH, Park N, Rundek T, Sacco RL (2005) Heritabilities of the metabolic syndrome and its components in the Northern Manhattan Family Study. Diabetologia 48:2006-2012

5. Freeman MS, Mansfield MW, Barrett JH, Grant PJ (2002) Heritability of features of the insulin resistance syndrome in a community-based study of healthy families. Diabet Med 19:994-999

6. Poulsen P, Vaag A, Kyvik K, Beck-Nielsen H (2001) Genetic versus environmental aetiology of the metabolic syndrome among male and female twins. Diabetologia 44:537-543

7. Teran-Garcia M, Bouchard C (2007) Genetics of the metabolic syndrome. Appl Physiol Nutr Metab 32:89-114

8. Zeggini E, Scott LJ, Saxena R et al (2008) Meta-analysis of genome-wide association data and large-scale replication identifies additional susceptibility loci for type 2 diabetes. Nat Genet 40:638-645 
9. Altshuler D, Hirschhorn JN, Klannemark M et al (2000) The common PPARgamma Pro12Ala polymorphism is associated with decreased risk of type 2 diabetes. Nat Genet 26:76-80

10. Frayling TM, Timpson NJ, Weedon MN et al (2007) A common variant in the FTO gene is associated with body mass index and predisposes to childhood and adult obesity. Science 316:889894

11. Gloyn AL, Weedon MN, Owen KR et al (2003) Large-scale association studies of variants in genes encoding the pancreatic beta-cell KATP channel subunits Kir6.2 (KCNJ11) and SUR1 (ABCC8) confirm that the KCNJ11 E23K variant is associated with type 2 diabetes. Diabetes 52:568-572

12. Grant SF, Thorleifsson G, Reynisdottir I et al (2006) Variant of transcription factor 7-like 2 (TCF7L2) gene confers risk of type 2 diabetes. Nat Genet 38:320-323

13. Sandhu MS, Weedon MN, Fawcett KA et al (2007) Common variants in WFS1 confer risk of type 2 diabetes. Nat Genet 39:951-953

14. Saxena R, Voight BF, Lyssenko V et al (2007) Genome-wide association analysis identifies loci for type 2 diabetes and triglyceride levels. Science 316:1331-1336

15. Scott LJ, Mohlke KL, Bonnycastle LL et al (2007) A genomewide association study of type 2 diabetes in Finns detects multiple susceptibility variants. Science 316:1341-1345

16. Sladek R, Rocheleau G, Rung J et al (2007) A genome-wide association study identifies novel risk loci for type 2 diabetes. Nature 445:881-885

17. Steinthorsdottir V, Thorleifsson G, Reynisdottir I et al (2007) A variant in CDKAL1 influences insulin response and risk of type 2 diabetes. Nat Genet 39:770-775

18. Zeggini E, Weedon MN, Lindgren CM et al (2007) Replication of genome-wide association signals in UK samples reveals risk loci for type 2 diabetes. Science 316:1336-1341

19. Barroso I, Luan J, Sandhu MS et al (2006) Meta-analysis of the Gly482Ser variant in PPARGC1A in type 2 diabetes and related phenotypes. Diabetologia 49:501-505

20. Bengtsson K, Melander O, Orho-Melander M et al (2001) Polymorphism in the beta(1)-adrenergic receptor gene and hypertension. Circulation 104:187-190

21. Dionne IJ, Garant MJ, Nolan AA et al (2002) Association between obesity and a polymorphism in the beta(1)-adrenoceptor gene (Gly389Arg ADRB1) in Caucasian women. Int J Obes Relat Metab Disord 26:633-639

22. Fujisawa T, Ikegami H, Kawaguchi Y, Ogihara T (1998) Metaanalysis of the association of Trp64Arg polymorphism of beta 3adrenergic receptor gene with body mass index. J Clin Endocrinol Metab 83:2441-2444

23. Jellema A, Zeegers MP, Feskens EJ, Dagnelie PC, Mensink RP (2003) Gly972Arg variant in the insulin receptor substrate-1 gene and association with type 2 diabetes: a meta-analysis of 27 studies. Diabetologia 46:990-995

24. Lange LA, Norris JM, Langefeld CD et al (2005) Association of adipose tissue deposition and beta-2 adrenergic receptor variants: the IRAS family study. Int J Obes (Lond) 29:449-457

25. McAteer JB, Prudente S, Bacci S et al (2008) The ENPP1 K121Q polymorphism is associated with type 2 diabetes in European populations: evidence from an updated meta-analysis in 42,042 subjects. Diabetes 57:1125-1130

26. Meyre D, Bouatia-Naji N, Tounian A et al (2005) Variants of ENPP1 are associated with childhood and adult obesity and increase the risk of glucose intolerance and type 2 diabetes. Nat Genet 37:863-867

27. Olivier M, Hsiung CA, Chuang LM et al (2004) Single nucleotide polymorphisms in protein tyrosine phosphatase 1beta (PTPN1) are associated with essential hypertension and obesity. Hum Mol Genet 13:1885-1892
28. Pihlajamaki J, Salmenniemi U, Vanttinen M et al (2006) Common polymorphisms of calpain-10 are associated with abdominal obesity in subjects at high risk of type 2 diabetes. Diabetologia 49:1560-1566

29. Wang H, Chu WS, Lu T, Hasstedt SJ, Kern PA, Elbein SC (2004) Uncoupling protein-2 polymorphisms in type 2 diabetes, obesity, and insulin secretion. Am J Physiol Endocrinol Metab 286:E1-E7

30. Weedon MN, Schwarz PE, Horikawa Y et al (2003) Meta-analysis and a large association study confirm a role for calpain-10 variation in type 2 diabetes susceptibility. Am J Hum Genet 73:1208-1212

31. Vimaleswaran KS, Luan J, Andersen G et al (2008) The Gly482Ser genotype at the PPARGC1A gene and elevated blood pressure: a meta-analysis of 13,949 individuals. J Appl Physiol doi:10.1152/japplphysiol.90423.2008

32. Widen E, Lehto M, Kanninen T, Walston J, Shuldiner AR, Groop LC (1995) Association of a polymorphism in the beta 3adrenergic-receptor gene with features of the insulin resistance syndrome in Finns. N Engl J Med 333:348-351

33. Lyssenko V, Jonsson A, Pulizzi N et al (2008) Clinical risk factors, DNA variants and the development of type 2 diabetes. $\mathrm{N}$ Engl J Med (in press)

34. National Heart, Lung, and Blood Institute (2001) National Cholesterol Education Program: Executive Summary of the Third Report of the National Cholesterol Education Program (NCEP) Expert Panel on Detection, Evaluation, and Treatment of High Blood Cholesterol In Adults (Adult Treatment Panel III). JAMA 285:2486-2497

35. World Health Organization (1999) Definition, diagnosis, and classification of diabetes mellitus and its complications. Report of a WHO consultation. Part 1: Diagnosis and classification of diabetes mellitus. Geneva: World Health Organization

36. Alberti KG, Zimmet P, Shaw J (2005) The metabolic syndromea new worldwide definition. Lancet 366:1059-1062

37. Orho-Melander M, Melander O, Guiducci C et al (2008) A common missense variant in the glucokinase regulatory protein gene (GCKR) is associated with increased plasma triglyceride and C-reactive protein but lower fasting glucose concentrations. Diabetes doi: $10.2337 / \mathrm{db} 08-0516$

38. Sparso T, Andersen G, Nielsen T et al (2008) The GCKR rs780094 polymorphism is associated with elevated fasting serum triacylglycerol, reduced fasting and OGTT-related insulinaemia, and reduced risk of type 2 diabetes. Diabetologia 51:70-75

39. Vaxillaire M, Cavalcanti-Proenca C, Dechaume A et al (2008) The common P446L polymorphism in GCKR inversely modulates fasting glucose and triglyceride levels and reduces type 2 diabetes risk in the DESIR prospective general French population. Diabetes 57:2253-2257

40. Salopuro T, Lindstrom J, Eriksson JG et al (2004) Common variants in beta2- and beta3-adrenergic receptor genes and uncoupling protein 1 as predictors of the risk for type 2 diabetes and body weight changes. The Finnish Diabetes Prevention Study. Clin Genet 66:365-367

41. Clausen JO, Hansen T, Bjorbaek C et al (1995) Insulin resistance: interactions between obesity and a common variant of insulin receptor substrate-1. Lancet 346:397-402

42. Lyssenko V, Sjogren M, Almgren P et al (2008) Genetic prediction of the metabolic syndrome. Diabetes Metab Syndr Clin Res Rev doi:10.1016/j.dsx.2008.07.002

43. Purcell S, Cherny SS, Sham PC (2003) Genetic Power Calculator: design of linkage and association genetic mapping studies of complex traits. Bioinformatics 19:149-150

44. Benyamin B, Sorensen TI, Schousboe K, Fenger M, Visscher PM, Kyvik KO (2007) Are there common genetic and environmental factors behind the endophenotypes associated with the metabolic syndrome? Diabetologia 50:1880-1888 
45. Lyssenko V, Lupi R, Marchetti P et al (2007) Mechanisms by which common variants in the TCF7L2 gene increase risk of type 2 diabetes. J Clin Invest 117:2155-2163

46. Genuth S, Alberti KG, Bennett P et al (2003) Follow-up report on the diagnosis of diabetes mellitus. Diabetes Care 26:3160 3167

47. Fredriksson R, Hagglund M, Olszewski PK et al (2008) The obesity gene, FTO, is of ancient origin, up-regulated during food deprivation and expressed in neurons of feeding-related nuclei of the brain. Endocrinology 149:2062-2071

48. Gerken T, Girard CA, Tung YC et al (2007) The obesityassociated FTO gene encodes a 2-oxoglutarate-dependent nucleic acid demethylase. Science 318:1469-1472

49. Lakka HM, Laaksonen DE, Lakka TA et al (2002) The metabolic syndrome and total and cardiovascular disease mortality in middle-aged men. JAMA 288:2709-2716
50. Chambers JC, Elliott P, Zabaneh D et al (2008) Common genetic variation near MC4R is associated with waist circumference and insulin resistance. Nat Genet 40:716-718

51. Loos RJ, Lindgren CM, Li S et al (2008) Common variants near MC4R are associated with fat mass, weight and risk of obesity. Nat Genet 40:768-775

52. Kathiresan S, Melander O, Guiducci C et al (2008) Six new loci associated with blood low-density lipoprotein cholesterol, highdensity lipoprotein cholesterol or triglycerides in humans. Nat Genet 40:189-197

53. Kooner JS, Chambers JC, Aguilar-Salinas CA et al (2008) Genome-wide scan identifies variation in MLXIPL associated with plasma triglycerides. Nat Genet 40:149-151

54. Willer CJ, Sanna S, Jackson AU et al (2008) Newly identified loci that influence lipid concentrations and risk of coronary artery disease. Nat Genet 40:161-169 